\title{
Vibration Based Gear Fault Diagnosis under Empirical Mode Decomposition and Power Spectrum Density Analysis
}

\author{
M. Ammar Akram 1*, Shahab Khushnood', Syeda Laraib Tariq', \\ Hafiz Muhammad Ali', Luqman Ahmad Nizam' \\ 1 Department of Mechanical Engineering, University of Engineering and Technology, Taxila, Pakistan \\ * Corresponding author's e-mail: mammarakram@gmail.com
}

\begin{abstract}
Rotating machinery plays a significant role in industrial applications and covers a wide range of mechanical equipment. A vibration analysis using signal processing techniques is generally conducted for condition monitoring of rotary machinery and engineering structures in order to prevent failure, reduce maintenance cost and to enhance the reliability of the system. Empirical mode decomposition (EMD) is amongst the most substantial non-linear and non-stationary signal processing techniques and it has been widely utilized for fault detection in rotary machinery. This paper presents the EMD, time waveform and power spectrum density (PSD) analysis for localized spur gear fault detection. Initially, the test model was developed for the vibration analysis of single tooth breakage of spur gear at different RPMs and then specific fault was introduced in driven gear under different damage conditions. The data, recorded by means of a wireless tri-axial accelerometer, was then analyzed using EMD and PSD techniques and the results were plotted. The results depicted that EMD algorithms are found to be more functional than the ordinarily used PSD and time waveform techniques.
\end{abstract}

Keywords: spur gears, tooth breakage, vibration amplitude, empirical mode decomposition, power spectrum density, time waveform.

\section{INTRODUCTION}

Rotary machines like industrial gearboxes, aircraft engines, power stations, etc. are an essential element of various mechanical systems. The transverse vibrations are produced due to fatigue, in many rotor elements of rotor dynamic systems, which can result in severe damage and catastrophic failure of the machinery. The stationary vibration signals are generated due to certain machine component defects like belt and pulley, rotor unbalance, looseness, shaft bow and cracks, rotor hub, misalignment, and coupling defects [1]. Wear, cracking, pitting, scuffing and spelling are frequently occurring gear damages, which induce complex transient vibration signals due to gearbox resonance, tooth meshing, gear and pinion shaft rotation. Multistage gearboxes vibrational signals are transient in nature because of impulses, high frequency faults and random noises [2].

Ordinary techniques, incorporating power spectrum and cepstrum estimation, crest factor, kurtosis, time-domain averaging, demodulation, have turned out to be successful in fault detection and are currently well-established. However, their major disadvantage is that they are based on presumption of stationarity and linearity of the signal generation process $[3 \div 7]$.

The developed signal processing techniques are being induced on vibration signals to detect, locate and identify faults and based on this, lifespan of a machine can also be estimated including neural network, genetic programming and algorithms, fuzzy logic, wavelet transform and machine support vectors [6]. Waveform analysis consists in recording data time history and is useful for non-steady conditions and short transient impulses analysis. 
Cooley and Tukey developed FFT algorithmic rule in 1965 which lessens the range of computations entailed for $\mathrm{N}$ points from $2 \mathrm{~N}^{2}$ to $2 \mathrm{~N} \log 2(\mathrm{~N})$, wherever $\log$ is that the base- 2 index. Spectral density is the Fourier Transform of auto correlation function. FFT analyzes the vibration signals with limited number of ascendant frequency components, whereas power spectral densities (PSD) are used for random vibration signals. PSD is governed by multiplying each frequency bin by its complex conjugate, in FFT, which ends up in the real spectrum of amplitude in $\mathrm{g}^{2}$. The fundamental purpose of PSD is to make it more proficient than an FFT for random vibration analysis, involving normalization of this amplitude value to the frequency bin width to obtain units of $\mathrm{g}^{2} / \mathrm{Hz}$. The vibration levels in signals of different lengths can be obtained by normalizing the results and hence, disposing of the dependency on bin width $[8 \div 10]$. The most significant time frequency analysis techniques for transient signal include Hilbert Huang Transform (HT), Short Time Fourier Transform (STFT), Continuous Wavelet Analysis (CWT), and Winger Ville Distribution (WVD).

In a STFT technique, windowing function is utilized to initially break the signal into different uniform segments and then FFT is applied on each segment of the signal. However, the major drawback of this technique is poor time frequency resolution and to overcome this problem, WVD evolved to strengthen the time frequency resolution. However, the main disadvantage of the technique is the generation of cross terms in the time frequency domain. Thus, for the further advancement in the time frequency resolution, the CWT technique is used $[11 \div 13]$. Haung et al. proposed a new time frequency analysis method, known as EMD, in 1998 to extricate all the oscillatory modes embedded in a signal without any requirement of data linearity. A signal is decomposed using EMD, into a limited sum of elements known as intrinsic mode functions (IMF) [14].

The time-frequency representation of Hilbert spectrum is achieved by the implementation of Hilbert transform to the IMFs. EMD is comparatively a far better technique than the other time and frequency domain techniques and finds application in numerous fields, such as nuclear physics [15], image processing [16], biomedical diagnostics [17], ocean and seismic engineering $[18,19]$ and structural testing [20]. EMD is used for mechanical and rotary machinery faults diagnosis such as beam crack detection [21], structural health monitoring [22], rolling bearings fault diagnosis [ $23 \div 25]$, gear fault diagnosis $[19,26 \div 27,28]$, rub signal analysis and rotor startup signal processing [29].

The functionality of EMD in the fault detection of spur gear was studied by Loutridis [19]. The energies of IMF were compared during the fault analysis which revealed high susceptibility to gear damage. Parey et al. [30] determined the gear fault severity performing both experimentation and simulation to authenticate the efficiency of EMD approach. The fault diagnostic information was enhanced by utilizing the statistical variables such as crest factor and kurtosis of the EMD signal.

Parey and Tandon [27] exhibited a systematic model, associating the vibration signal to the defect size on the flank of gear tooth. Defect development was accessed utilizing kurtosis and rms values of the intrinsic modes of the disintegrated vibration signals. Kurtosis of the EMD signal depicted better affectability contrasted with vibration signal kurtosis for the fault seriousness. However, EMD is characterized by certain drawbacks, involving recurring appearance of mode mixing, which can be overcome by using ensemble empirical mode decomposition (EEMD) to minimize this effect. EMD perhaps over-decays a signal due to the noises effect in a way that few intrinsic mode functions are deformed and are unable to symbolize the signal features. Combined mode function (CMF) was recommended to sort out this problem. EMD is an adaptive filter bank in its nature, whereas the CMF method redesigns the filter bank [31, 32]. The objective of this paper is to analyze the vibrations produced by various faults of gear. The results depict better fault diagnostic information in spur gears transference system by utilizing the suggested methods, thereby enhancing the condition monitoring potentiality of the designed geared system.

\section{EMD BASIC ALGORITHM}

The EMD method decays a time-series into a limited set of oscillatory functions known as IMF. An IMF function assures the following conditions:

- The number of zero crossings and the number of extrema should either be equal or differ at most by one;

- At a certain point, the running mean value of the envelopes is zero, defined by the local maxima and the local minima. 
Within a signal, the IMFs indicate the simple oscillation mode where, EMD is a "sifting" operation, attuned to extricate the IMFs by the steps illustrated below:

Firstly, $m_{1}$ is consigned as the two envelopes mean, and $h_{1}$ as the contrast between the signals $x(t)$ and $m_{1}$, which is taken as the first component, i.e.

$$
x(t)-m_{1}=h_{1}
$$

Then disparate the first IMF $c_{1}$ from $x(t)$ by

$$
x(t)-c_{1}=r_{1}
$$

Use residue $r_{1}$ as the original signal and then put it through the same process as above, so that we can achieve other IMFs, $c_{1}, c_{2}, c_{3}, \ldots ., c_{n}$, which yields

$$
\left\{\begin{array}{l}
r_{1}-c_{2}=r_{2} \\
\cdots \ldots \ldots \ldots \\
r_{n-1}-c_{n}=r_{n}
\end{array}\right.
$$

The signal $x(t)$ is then disintegrated into $n$ intrinsic modes and a residue $r_{n}$. Figure 1 elaborates the flow diagram of EMF algorithm.

\section{EXPERIMENTAL TEST RIG}

The tests were conducted on a single-stage spur gear using a single phase induction motor with the output of $0.5 \mathrm{HP}$, which is running at variable RPMs speed i.e. 1386 and $1462 \mathrm{rpm}$ along with a variable frequency driver to vary the speed of the system. Horizontal milling machine with a module of 2 was used to fabricate the gears.

The gear system has two parallel gears having low speed gear with 12 teeth number and the pinion teeth number is 36 . The highspeed shaft pinion is faulty gear in the test rig. The defect was

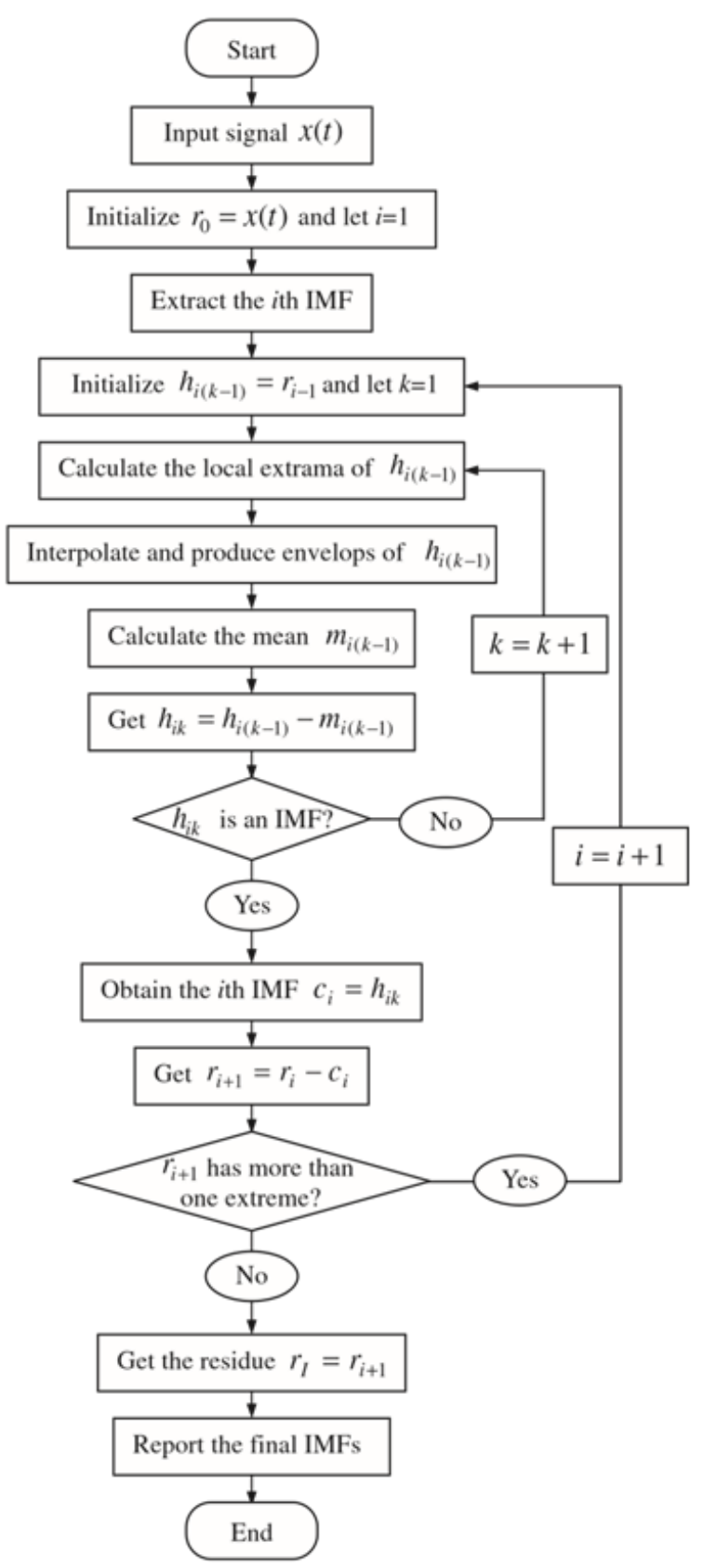

Fig. 1. EMD Flow Chart [33]

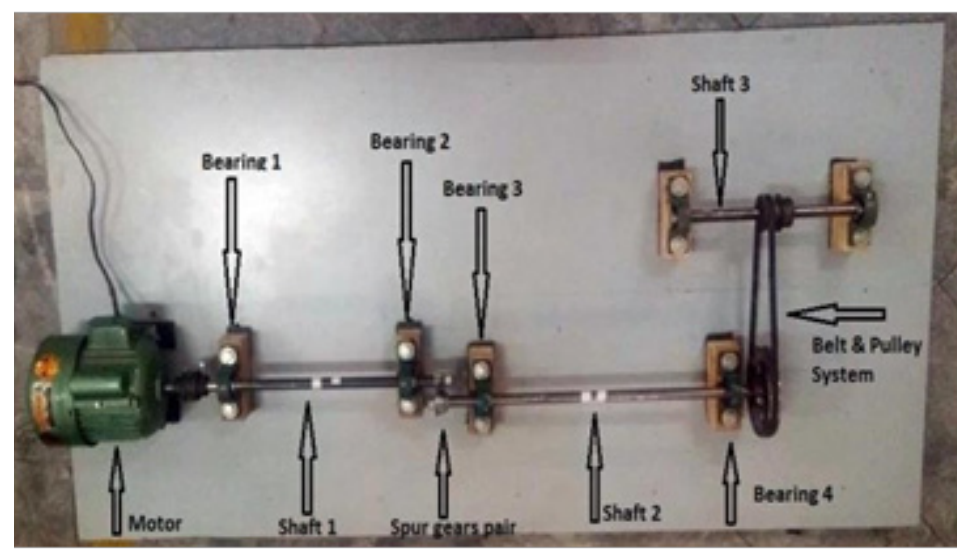

Fig. 2. Experimental test rig with gears and pulley system [34] 
induced by eliminating one tooth of the spur gear with percentage of $25,50,75$ and $100 \%$, respectively. Figure 2 shows the experimental test rig.

G-Link tri-axial accelerometer, developed by MICROSTRAIN Corp. was used to gather the data for the vibration analysis for horizontal, vertical and axial direction. The readings acquired from accelerometer were evaluated using SIGVIEW and MATLAB software. Figure 3 shows different stages of data collection and analysis.

\section{EXPERIMENTAL RESULTS}

\section{Time waveform analysis}

The most powerful and conventional technique to diagnose gear faults is the time waveform. Following are the time waveform graphs for 25,75 and $100 \%$ for 1462 RPM for first tooth breakage and it can be observed that an increase in the amplitude of the generated impacts has a direct relation with the increase in the intensity of a fault. Upon each revolution there will be one impact if one tooth is defected and two impacts per revolution in case if two teeth are defected.

It is clear from the graphs that for $25 \%$ of tooth breakage, the impulse amplitude is small, and it occurred once per revolution. As the intensity of fault impulse grows, the amplitude increased up to $100 \%$ of tooth removal; hence, the

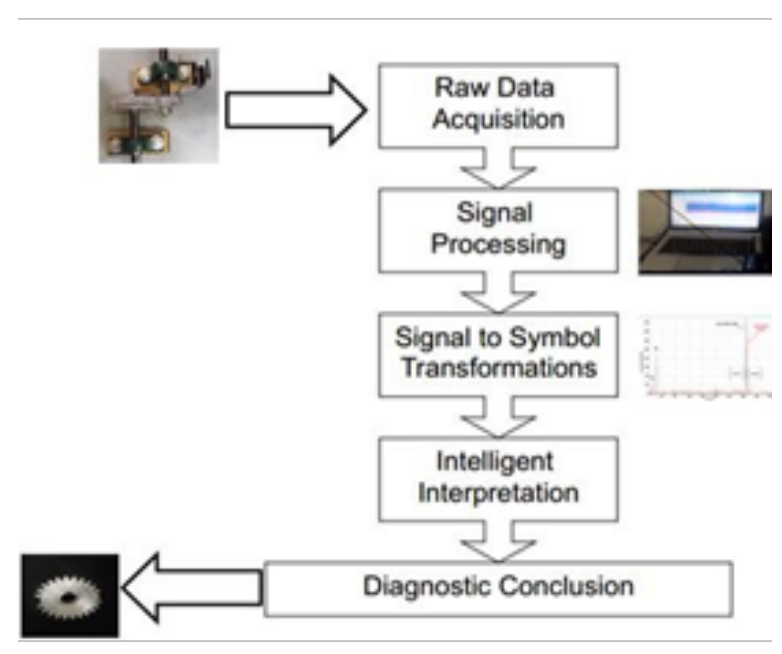

Fig. 3. Stages of data collection and analysis [34]

amplitude will be at maximum. Similarly, in case of increased tooth damage, the time waveform will show multiple impulses per revolution.

\section{Power spectrum density analysis}

The gearbox vibration signals are casually noisy and periodic. The PSD technique showed a much better fault identification. The experiment was conducted for variable speeds of 1386 and 1462 RPM for a broken tooth and the results are illustrated in Figure 5. For the corresponding RPM, we have Gear Rotational Frequency (GRF) $\left(\mathrm{X}_{\mathrm{g}}=11.55 \mathrm{~Hz}, 12.18 \mathrm{~Hz}\right.$ ), Gear Pinion Frequency $(\mathrm{GPF})\left(\mathrm{X}_{\mathrm{p}}=23.1 \mathrm{~Hz}, 24.36 \mathrm{~Hz}\right)$ and Gear Mesh

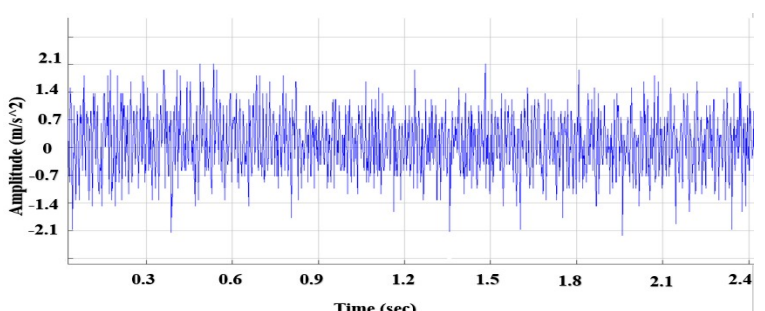

(a)

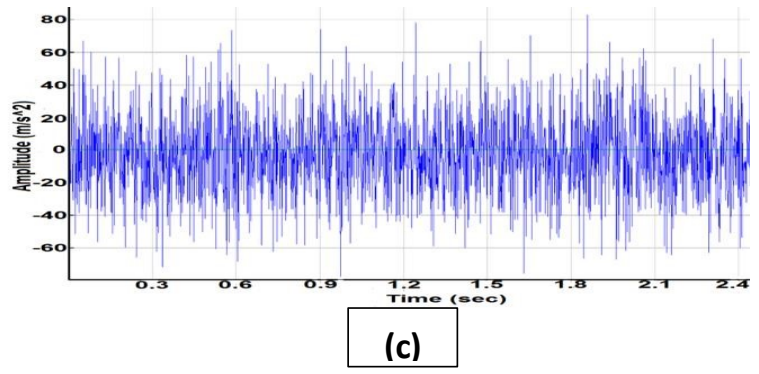

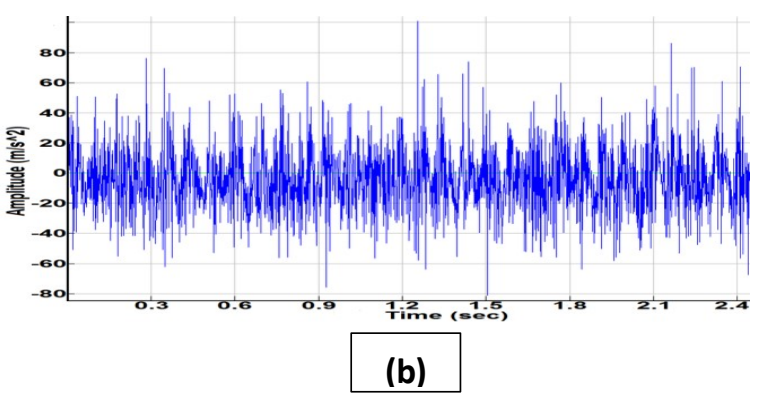

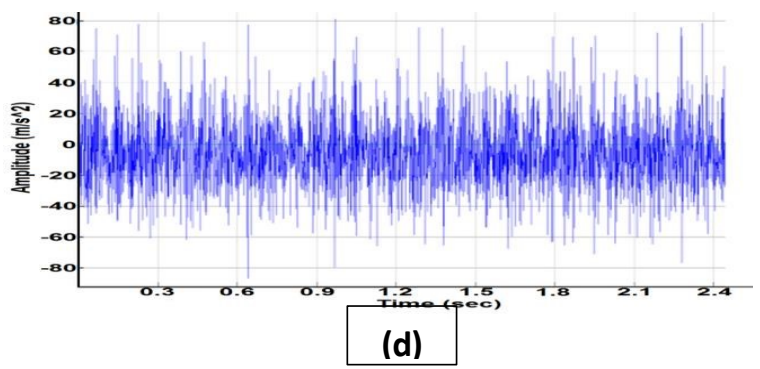

Fig. 4. Time domain plots for first tooth breakage percentage of (a) $0 \%$ (b) $25 \%$ (c) $75 \%$ (d) $100 \%$ 


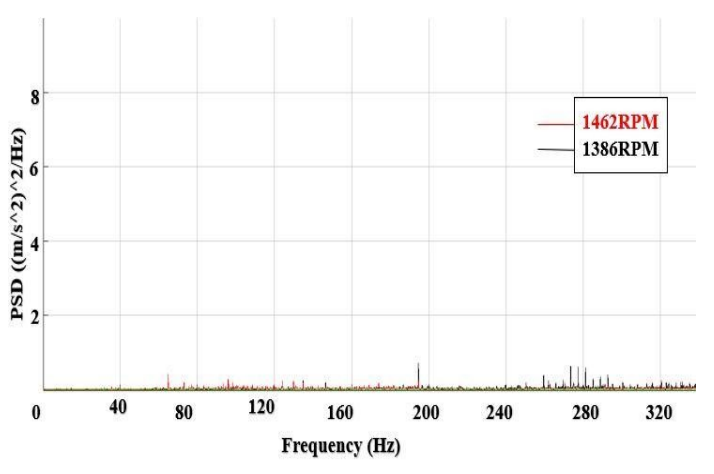

(a)

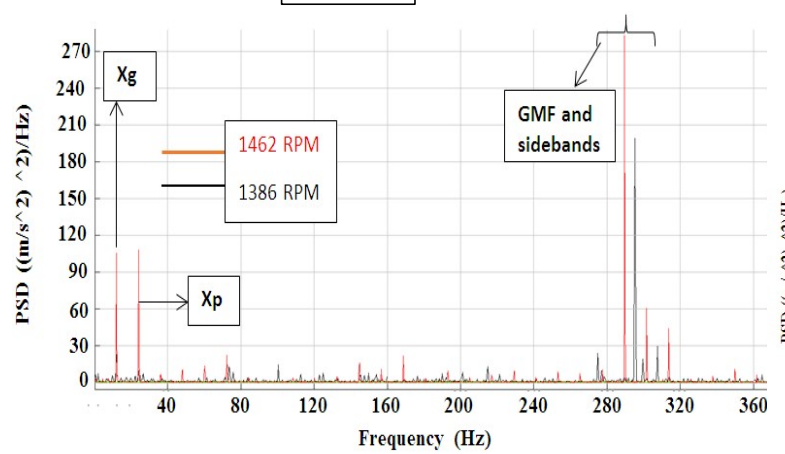

(c)

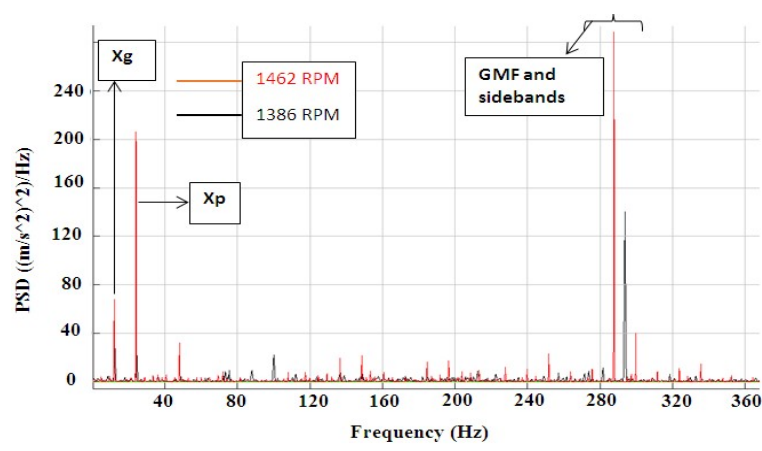

(b)

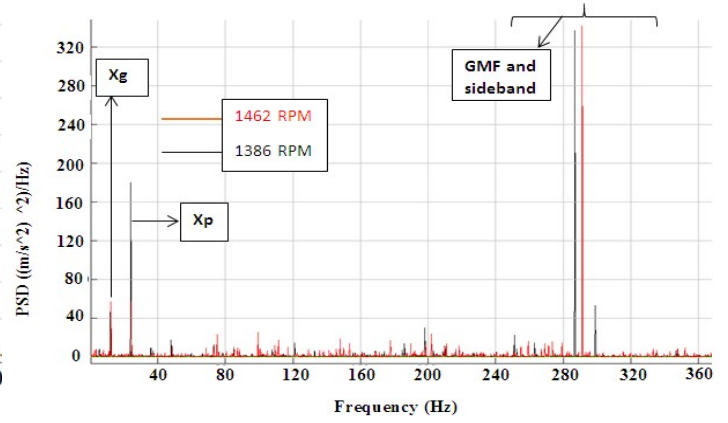

(d)

Fig. 5. PSD plots for tooth breakage percentage of (a) $0 \%$ (b) $25 \%$ (c) $75 \%$ (d) $100 \%$

frequency $(\mathrm{GMF}=277.2 \mathrm{~Hz}, 292.8 \mathrm{~Hz})$. The amplitude of GMF, GNF, GPF increases up to $75 \%$ of tooth breakage and then decays upon complete tooth removal whereas, sidebands level will enhance throughout with fault intensity.

Gear Natural Frequency (GNF) also rises in its amplitude with an increase in fault severity, thus causing the instability in the system. The results depicted that sidebands occurred due to tooth breakage are more sensitive as compared to the Gear Mesh Frequency (GMF). That means when there is no tooth, there will be less vibration from it depending upon the teeth mesh, but sidebands will predominantly increase in amplitude [30,35]. Figure 5 shows the Fourier transform zoomed in plot, which indicates numerous sidebands around the specified gear mesh frequency along with its second and third harmonics for both speeds. Remarkably, the existence of sidebands of gear rotational frequency at $9.4 \mathrm{~Hz}$ for 1464 RPM around the gear mesh frequency with its harmonic components is near to the gear rotational frequency. This approximates the viable subsistence of a confined gear defect.

\section{Empirical mode decomposition analysis}

Five IMFs (IMF1 to IMF5) with residual were enumerated at 1464 RPM for different gear tooth fault intensities and the corresponding PSD spectrum was contemplated to extract diagnostic information.

Figure 6 (a) and 6 (b), indicate the neighboring impulses average time spacing is about $0.003420 \mathrm{~s}$ or $292.397 \mathrm{~Hz}$, indicating damaged gear meshing frequency. Transient frequencies range from 200 $\mathrm{Hz}$ to about $320 \mathrm{~Hz}$, including sidebands of the meshing frequency of the gear pair corresponding to transmission path. The indicated features confirm the existence of a localized defect on the gear pair pinion. From the results of EMD decomposition in Figs. 6 and 7, the IMF1 is mainly a highfrequency component, which is predominantly between $120 \mathrm{~Hz}$ and $980 \mathrm{~Hz}$ and is mainly composed of modulating the gear mesh frequency and its side band. IMF2 comes from the high-speed shaft gear meshing and its frequency belongs to the interference component. IMF3 to IMF7 are low-frequency components, including generator frequency and some other interference frequency. Therefore, the 

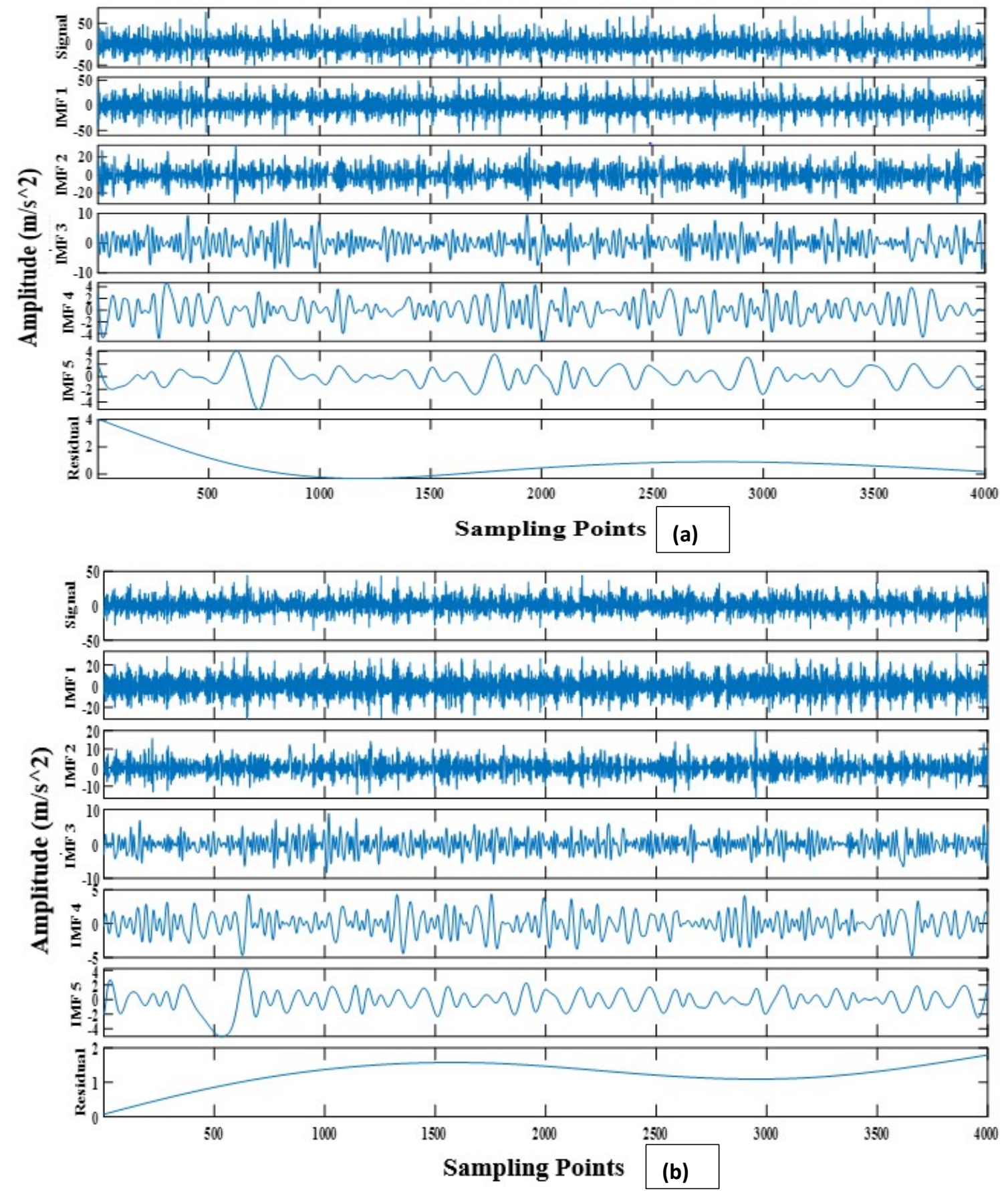

Fig. 6. Gear break fault signal EMD decomposition results for tooth percentage (a) $25 \%$ (b) $75 \%$

fault characteristics of the middle speed pinion are mainly concentrated in the three signals of IMF1, IMF2 and IMF3 [26, 36, 37].

Figure 7 elaborates why the frequency spectrum of the signal using EMD has performed better than PSD. Fig. 7 (c) indicates that the generated sidebands by impact overlap the rotating frequency spectrum lines of driver shaft and the half rotating frequency of driven shaft because of half reduction gear pair mechanism. It is challenging to diagnose the fault by extracting the fault characteris- tics from spectrum. EMD is an adaptive band-pass filter bank as mentioned earlier, the bandwidth of which is determined by the local characteristic time scales signal features. In addition to EMD Adaptive filter bank, CMF approach can also be utilized to the spectrum lines and sideband of the driver and driven shaft, respectively $[33,38]$.

However, comparing the analyzing results of using Time waveform (Fig. 4), PSD (Fig. 5), and EMD (Fig. 6 and Fig. 7), EMD can extract the fault characteristics from this signal more effectively. 

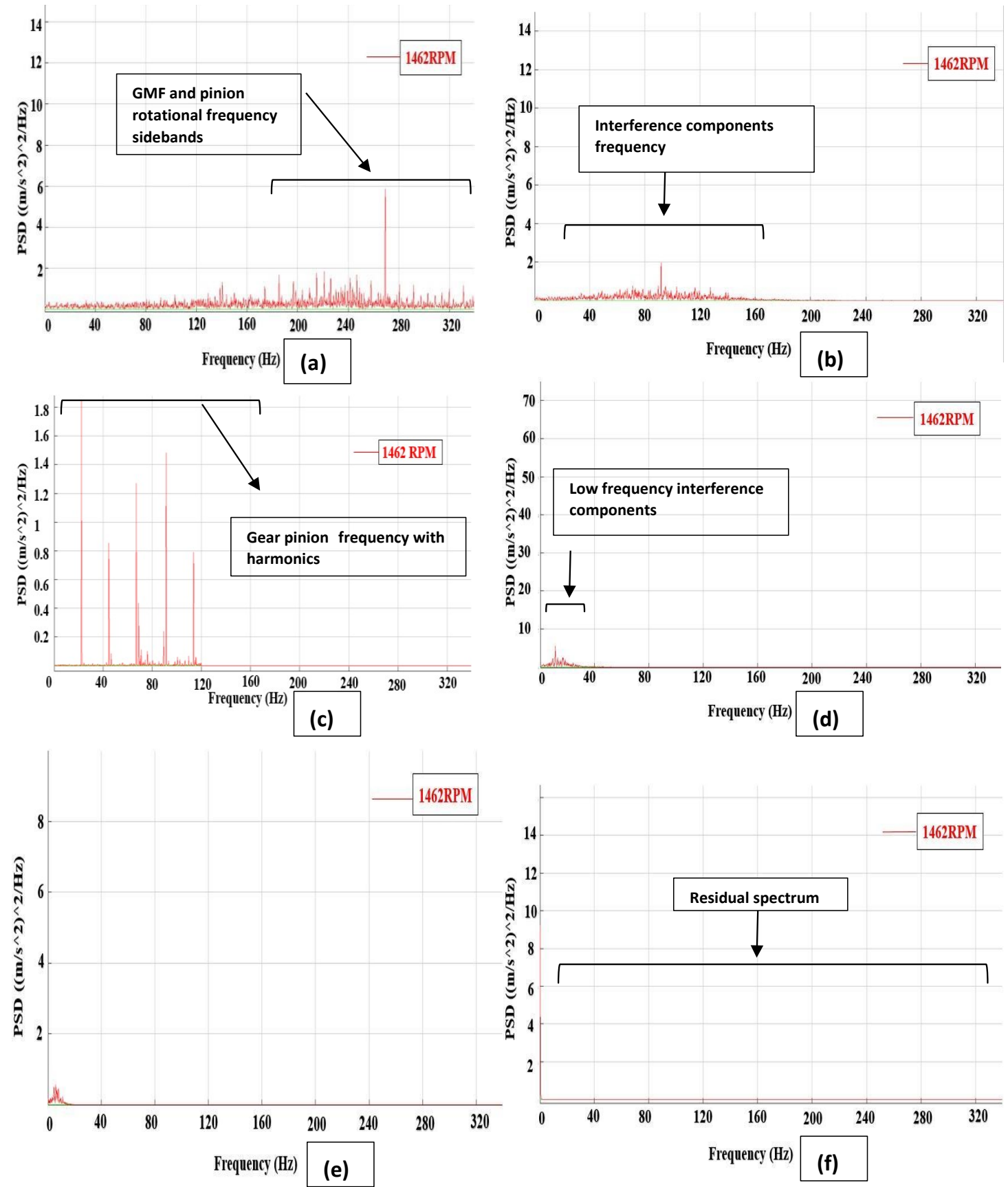

Fig. 7. Spectrum of IMF components (a) IMF1

(b) IMF2 (c) IMF3 (d) IMF4 (e) IMF5 (f) Residual

\section{CONCLUSION}

The empirical mode decomposition dispenses an advanced method for time-frequency evaluation in non-stationary vibration signal and attracted great attention in different areas. In this paper, we implemented this method for localized spur gear fault diagnosis. It was revealed that the EMD is more likely to enhance the transients excited by the broken tooth than the regularly used time waveform and power spectrum density examination.

\section{Acknowledgements}

This work is encouraged by Mechanical Engineering Department, UET Taxila to carry out the research. We thank Muhammad Kamran Akram from Vàr Energi for financial support. Special thanks to Andreas Ederer and Zeeshan Kareem from Baumüller Services Nuremberg, Germany and Electrical Engineering Department for providing technical guidance for conduction of experiment. 


\section{REFERENCES}

1. J. Xuan, H. Jiang, T. Shi, and G. Liao, Gear fault classification using genetic programming and support vector machines, Int. J. Inf. Technol, vol. 11, no. 9, p. 37, 2005.

2. V. Karma and H. Borade, Fault diagnosis of single stage spur gearbox using narrow band demodulation technique: Effect of spalling, Int. J. Res. Eng. Technol., vol. 1, no. 3, pp. 11-16, 2013.

3. R.M. Stewart, Some useful data analysis techniques for gearbox diagnostics, University of Southampton Report MHM, R/10/77, July, 1977.

4. R.B. Randall, Advances in the application of cepstrum analysis to gearbox diagnosis, in 2nd Int Conf Vib Rotating Mach 1980, 1980, pp. 169-174.

5. P.D. McFadden and J. D. Smith, A signal processing technique for detecting local defects in a gear from the signal average of the vibration, Proc. Inst. Mech. Eng. Part C J. Mech. Eng. Sci., vol. 199, no. 4, pp. 287-292, 1985.

6. W. J. Staszewski, K. Worden, and G. R. Tomlinson, Time-frequency analysis in gearbox fault detection using the Wigner-Ville distribution and pattern recognition, Mech. Syst. Signal Process., vol. 11, no. 5, pp. 673-692, 1997.

7. R. B. Randall, A new method of modeling gear faults, J. Mech. Des., vol. 104, no. 2, pp. 259-267, 1982.

8. W. Lai, J. Xuan, T. Shi, and S. Yang, Research of Vigner-Ville Time Frequency and Application in Detecting Gear Pinion Fault, J. Vib. Eng., vol. 16, no. 2, pp. 247-250, 2003.

9. J. Hanna and H. Luo, Wind turbine gearbox planetary stage gear damage detection using vibration data, in ASME Turbo Expo 2014: Turbine Technical Conference and Exposition, 2014, p. V03BT46A014-V03BT46A014.

10. J.D. Smith, Gears and their vibration: a basic approach to understanding gear noise, Marcel Dekker, Inc, 270 Madison Ave, New York, N. Y. 10016, 1983. 170, 1983.

11. X. Fan and M. J. Zuo, Gearbox fault detection using Hilbert and wavelet packet transform, Mech. Syst. Signal Process., vol. 20, no. 4, pp. 966-982, 2006.

12. W.J. Wang and P. D. McFadden, Early detection of gear failure by vibration analysis $i$. calculation of the time-frequency distribution, Mech. Syst. Signal Process., vol. 7, no. 3, pp. 193-203, 1993.

13. W.J. Wang and P. D. McFadden, Application of wavelets to gearbox vibration signals for fault detection, J. Sound Vib., vol. 192, no. 5, pp. 927-939, 1996.

14. N.E. Huang et al., The empirical mode decomposition and the Hilbert spectrum for nonlinear and non-stationary time series analysis, Proc. R. Soc.
London. Ser. A Math. Phys. Eng. Sci., vol. 454, no. 1971, pp. 903-995, 1998.

15. M.E. Montesinos, J. L. Munoz-Cobo, and C. Perez, Hilbert-Huang analysis of BWR neutron detector signals: application to DR calculation and to corrupted signal analysis, Ann. Nucl. Energy, vol. 30, no. 6, pp. 715-727, 2003.

16. J.C. Nunes, Y. Bouaoune, E. Delechelle, O. Niang, and $\mathrm{P}$. Bunel, Image analysis by bidimensional empirical mode decomposition, Image Vis. Comput., vol. 21, no. 12, pp. 1019-1026, 2003.

17. R. Balocchi et al., Deriving the respiratory sinus arrhythmia from the heartbeat time series using empirical mode decomposition, Chaos, Solitons \& Fractals, vol. 20, no. 1, pp. 171-177, 2004.

18. A.D. Veltcheva, Wave and group transformation by a Hilbert spectrum, Coast. Eng. J., vol. 44, no. 4, pp. 283-300, 2002.

19. S.J. Loutridis, Damage detection in gear systems using empirical mode decomposition, Eng. Struct., vol. 26, no. 12, pp. 1833-1841, 2004.

20. S.T. Quek, P. S. Tua, and Q. Wang, Detecting anomalies in beams and plate based on the Hilbert-Huang transform of real signals, Smart Mater. Struct., vol. 12, no. 3, p. 447, 2003.

21. S. Loutridis, E. Douka, and L. J. Hadjileontiadis, Forced vibration behaviour and crack detection of cracked beams using instantaneous frequency, $\mathrm{Ndt}$ E Int., vol. 38, no. 5, pp. 411-419, 2005.

22. D. Pines and L. Salvino, Structural health monitoring using empirical mode decomposition and the Hilbert phase, J. Sound Vib., vol. 294, no. 1-2, pp. 97-124, 2006.

23. Y. Yu and C. Junsheng, A roller bearing fault diagnosis method based on EMD energy entropy and ANN, J. Sound Vib., vol. 294, no. 1-2, pp. 269277, 2006.

24. D. Yu, J. Cheng, and Y. Yang, Application of EMD method and Hilbert spectrum to the fault diagnosis of roller bearings, Mech. Syst. Signal Process., vol. 19, no. 2, pp. 259-270, 2005.

25. Q. Gao, Z. J. He, X. F. Chen, and K. Y. Qi, Detecting damage of rolling bearings using EMD, in Key Engineering Materials, 2005, vol. 293, pp. 753-760.

26. B. Liu, S. Riemenschneider, and Y. Xu, Gearbox fault diagnosis using empirical mode decomposition and Hilbert spectrum, Mech. Syst. Signal Process., vol. 20, no. 3, pp. 718-734, 2006.

27. A. Parey and N. Tandon, Impact velocity modelling and signal processing of spur gear vibration for the estimation of defect size, Mech. Syst. Signal Process., vol. 21, no. 1, pp. 234-243, 2007.

28. A. Parey, M. El Badaoui, F. Guillet, and N. Tandon, Dynamic modelling of spur gear pair and application of empirical mode decomposition-based 
statistical analysis for early detection of localized tooth defect, J. Sound Vib., vol. 294, no. 3, pp. 547-561, 2006.

29. G. Gai, The processing of rotor startup signals based on empirical mode decomposition,"Mech. Syst. Signal Process., vol. 20, no. 1, pp. 222235, 2006.

30. S. Wu, M. J. Zuo, and A. Parey, Simulation of spur gear dynamics and estimation of fault growth, J. Sound Vib., vol. 317, no. 3-5, pp. 608-624, 2008.

31. Y. Lei, Z. He, and Y. Zi, Application of the EEMD method to rotor fault diagnosis of rotating machinery, Mech. Syst. Signal Process., vol. 23, no. 4, pp. 1327-1338, 2009.

32. M. Žvokelj, S. Zupan, and I. Prebil, Multivariate and multiscale monitoring of large-size low-speed bearings using ensemble empirical mode decomposition method combined with principal component analysis, Mech. Syst. Signal Process., vol. 24, no. 4, pp. 1049-1067, 2010.

33. Y. Lei, J. Lin, Z. He, and M. J. Zuo, A review on empirical mode decomposition in fault diagnosis of rotating machinery, Mech. Syst. Signal Process., vol. 35, no. 1-2, pp. 108-126, 2013.

34. H. M. Ali et al., Experimental Analysis of Tooth Breakage Effect on the Vibration Characteristics of Spur Gears, Tech. J., vol. 23, no. 1, pp. 42-52, 2018.

35. M. Lebold, K. McClintic, R. Campbell, C. Byington, and K. Maynard, Review of vibration analysis methods for gearbox diagnostics and prognostics, in Proceedings of the 54th meeting of the society for machinery failure prevention technology, 2000, vol. 634, p. 16.

36. Q. Gao, C. Duan, H. Fan, and Q. Meng, Rotating machine fault diagnosis using empirical mode decomposition, Mech. Syst. Signal Process., vol. 22, no. 5, pp. 1072-1081, 2008.

37. D. Han, N. Zhao, and P. Shi, Gear fault feature extraction and diagnosis method under different load excitation based on EMD, PSO-SVM and fractal box dimension, J. Mech. Sci. Technol., vol. 33, no. 2, pp. 487-494, 2019.

38. R. Ricci and P. Pennacchi, Diagnostics of gear faults based on EMD and automatic selection of intrinsic mode functions, Mech. Syst. Signal Process., vol. 25, no. 3, pp. 821-838, 2011. 